\title{
Um modelo conceitual para a gamificação de Ambientes Virtuais de Aprendizagem
}

\author{
Ana Carolina Tomé Klock - actklock@gmail.com \\ Lucas Felipe da Cunha - lucasfelipedacunha@gmail.com \\ Isabela Gasparini - isabela.gasparini@udesc.br \\ Departamento de Ciência da Computação - PPGCA e PPGECMT \\ Universidade do Estado de Santa Catarina (UDESC) - Joinville - SC
}

\begin{abstract}
Resumo. Esse artigo apresenta um modelo conceitual para auxiliar na implementação da gamificação em ambientes virtuais de aprendizagem, observando os dados envolvidos, as tarefas disponíveis, os alunos que utilizam e os objetivos da gamificação. São levantadas diferentes características dos alunos que podem influenciar no sucesso da gamificação e frameworks existentes que auxiliam a fundamentar o modelo proposto. Por fim, o modelo proposto é aplicado a um ambiente virtual de aprendizagem.
\end{abstract}

Palavras-chave: gamificação, modelo conceitual, educação, AVA, adaptabilidade.

\section{A conceptual model for the gamification of Virtual Learning Environments}

\begin{abstract}
This paper presents a conceptual model to assist in the implementation of gamification in virtual learning environments, based on the data involved, the available tasks, the students who are using it and the goals of gamification. Different student characteristics that may influence the success of gamification are investigate and existing frameworks that may support the proposed model are raised. Finally, the model is applied to a virtual environment learning.
\end{abstract}

Keywords: gamification, conceptual model, education, VLE, adaptability.

\section{Introdução}

Atualmente a área de informática na educação tem buscado diferentes tecnologias que possam ser aplicadas para auxiliar nos processos de ensino e de aprendizagem. Diversas ferramentas têm sido exploradas, tais como o uso tecnologias vestíveis, tablets, learning analytics e MOOCs (cursos massivos gratuitos e on-line) (JOHNSON et al., 2013). Uma das tecnologias que tem sido amplamente estudadas e aplicadas na área educacional para motivar e engajar os alunos é a gamificação.

A gamificação é a utilização de técnicas existentes em jogos para motivar e engajar pessoas com objetivos da vida real. Para auxiliar o aluno a alcançar seu objetivo (aprender), a gamificação pode aumentar progressivamente a dificuldade dos exercícios, permitir que o aluno cometa erros (o que não é bem tolerado na educação tradicional) e promover competições e colaboração entre os alunos a fim de ensinar ao mesmo tempo em que diverte (FARDO, 2013). Entretanto, para que a gamificação produza o efeito esperado, é necessário que ela considere o conteúdo a ser aprendido, as características dos alunos e as tarefas disponíveis no ambiente virtual de aprendizagem.

Esse artigo propõe um modelo conceitual para auxiliar na implementação da gamificação nos ambientes virtuais de aprendizagem (AVAs), explicando sua aplicação em um ambiente virtual de aprendizagem. Para isso, a seção 2 expõe os conceitos principais envolvidos na gamificação e a seção 3 explica como as características dos alunos podem influenciar no sucesso da gamificação. Em seguida, a seção 4 explora os trabalhos relacionados que serviram para auxiliar no modelo proposto na seção 5 . A seção 6 demonstra como esse modelo conceitual foi aplicado e a seção 7 apresenta as conclusões do artigo.

\section{Fundamentos sobre Gamificação}

A gamificação (do inglês Gamification) é o termo utilizado para definir a utilização de elementos e design de jogos em contextos não lúdicos a fim de estimular e motivar as 
pessoas a alcançarem algum objetivo específico, como uma mudança de comportamento ou o desenvolvimento de novas habilidades (WERBACH; HUNTER, 2012; DETERDING et al., 2011a; BURKE, 2011). Apesar de o termo gamificação ter sido inventado em 2002 e de existirem pesquisas relacionadas aos benefícios que podem ser extraídos de jogos desde 1982 (como Malone (1982) e Draper (1999)), o tema é relativamente novo e só ganhou destaque a partir de 2010 (BURKE, 2014).

Entre os elementos de jogos comumente utilizados em ambientes virtuais de aprendizagem, podem-se citar: feedback constante, desafios, competição, conquistas, recompensas, pontos, medalhas, missões, personalização, regras, narrativa, níveis e rankings (BISSOLOTTI; NOGUEIRA; PEREIRA, 2014; KLOCK et al., 2014). A maioria desses elementos está diretamente relacionada com os desejos humanos, por exemplo, os pontos satisfazem a necessidade de recompensa, os níveis servem para demonstrar a posição do jogador entre os demais, os desafios permitem conquistar medalhas e os rankings estimulam a competição (BBVA INNOVATION EDGE, 2012). $\mathrm{O}$ atendimento dessas necessidades humanas pela gamificação trazem boas experiências para o aluno, como a satisfação de uso e o engajamento (DETERDING et al., 2011b).

Em um mesmo ambiente educacional, diferentes alunos, com características distintas, podem estar envolvidos em uma mesma disciplina. Essas características (por exemplo, pessoais, pedagógicas, culturais), podem fazer com que o aluno aprenda melhor de uma forma do que de outra. Portanto, é necessário entender os usuários para que a gamificação realmente traga os benefícios para a área educacional. A próxima seção apresenta uma análise sobre os diferentes tipos de usuários descritos na literatura.

\section{Análise dos tipos de usuários em ambientes gamificados}

Cada usuário é singular, seja por suas características individuais, pelo seu estilo cognitivo de aprendizagem, pelo seu comportamento durante os jogos ou ainda por questões culturais nas quais ele está envolvido.

Um estudo realizado por Pedro et al. (2015) com alunos da sétima série demonstrou que a utilização de elementos de jogos como pontos, medalhas, feedback constante, desafios e ranking em um ambiente virtual de aprendizagem melhorou o desempenho dos alunos do sexo masculino, mas piorou o desempenho das alunas do sexo feminino em comparação com os alunos e alunas que utilizavam o ambiente sem gamificação. Isso indica que a diferença entre o sexo dos usuários pode influenciar no sucesso da gamificação.

Outro estudo realizado por Codish e Ravid (2014) durante um semestre com alunos do curso de graduação analisa a diferença que o ambiente gamificado tem entre pessoas extrovertidas e introvertidas. Segundo Codish e Ravid (2014), foi possível constatar que as medalhas, recompensas e pontos motivaram mais os alunos extrovertidos do que os introvertidos durante o experimento.

Uma análise feita por Richard Bartle (1996) categorizou os tipos de jogadores conforme o que os motiva a jogar: conquistadores, socializadores, exploradores e assassinos. Os 'achievers' (ou conquistadores), também chamados de "ouros" (em alusão a um baralho de cartas), criam objetivos no jogo e tentam vigorosamente alcançá-los, o que implica que esse tipo de jogador fica mais motivado por acumular pontos e ascender níveis (BARTLE, 1996). Os 'socializers' (ou socializadores), também chamados de "copas", são jogadores que utilizam o jogo para conversar e interagir com seus colegas, motivando-se mais por ferramentas de comunicação e comandos entre jogadores, como presentear um colega no jogo (BARTLE, 1996). Os 'explorers' (ou exploradores), também chamados de "espadas", estão interessados em conhecer todos 
os truques e locais do jogo e a diversão para esse tipo de jogador está na descoberta, por isso é importante colocar desafios ocultos nos jogos para mantê-los engajados (BARTLE, 1996). Já os 'killers' (ou assassinos), também chamados de "paus", precisam se impor sobre os demais jogadores para se sentirem realizados e elementos como rankings e tabelas de classificação auxiliam a mantê-los motivados (BARTLE, 1996). Apesar de cada tipo de jogador ser diferente dos demais, os tipos não são mutuamente exclusivos e o usuário pode ser mais parecido com um dos tipos ou ser uma mescla de alguns ou todos eles (ZICHERMANN; LINDER, 2010).

Outro estudo realizado por Almaliki, Ncube e Ali (2014) compara as diferenças culturais de pessoas do Oriente Médio (Arábia Saudita, Irã e Egito) e de pessoas da Europa (Países Baixos, Reino Unido e Espanha). Almaliki et al. (2014) comentam que as pessoas do Oriente Médio consideram o feedback mais importante e o uso de elementos como medalhas, personalização e a possibilidade de descoberta de itens escondidos irão motivar mais pessoas do Oriente Médio do que da Europa.

Ao identificar quem são os usuários e suas preferências, é possível incluir os elementos de jogos que atraiam diferentes tipos de usuários de acordo com o público alvo (HÄGGLUND, 2012). Hägglund (2012) também explica que, ao conhecer os usuários, ou pelo menos aplicar um elemento que motive cada tipo de usuário, todos os tipos terão algo para se motivarem e isso aumentará a chance de atração, uma vez que é difícil (senão impossível) agradar a todos os usuários/alunos.

\section{Trabalhos relacionados}

Para entender o processo de criação de um modelo conceitual e quais características devem ser consideradas, foi realizada uma pesquisa de diferentes modelos conceituais projetados para ajudar na aplicação de gamificação.

O primeiro modelo conceitual analisado foi proposto por Chou (2013) e é chamado de "Octalysis", pois ele é dividido em oito núcleos diferentes. Cada núcleo é responsável pela análise de características dos usuários que Chou (2013) considera relevante para a gamificação do ambiente. Um dos núcleos importantes para o entendimento desse trabalho é o núcleo do "Desenvolvimento e Realização", que é responsável pelos desafios para cada usuário no ambiente, tal como progresso e o desenvolvimento de habilidades dos usuários (CHOU, 2013). Quando esse núcleo é ativado, os usuários são guiados para alcançar algum objetivo através de elementos como pontos, níveis, rankings, conquistas e medalhas. Outro núcleo interessante é o núcleo da "Influência Social e Afinidade", que é responsável por todos os tipos de elementos sociais que fazem parte do sistema, baseando-se no desejo das pessoas de se conectarem e se compararem com os demais (CHOU, 2013). Chou (2013) explica que esse núcleo pode ser o que mais mantém os usuários engajados e motivados se utilizado corretamente, uma vez que os usuários estão mais dispostos a investir o tempo delas no ambiente se souberem que seus amigos também investem o tempo deles. Este modelo conceitual proposto não é específico para ambientes educacionais, podendo ser aplicado a diferentes domínios.

O segundo modelo analisado foi criado por Marache-Francisco e Brangier (2013) e divide o processo de gamificação em duas etapas: análise de contexto e design interativo. A etapa de análise de contexto é um pré-requisito para entender as intenções e o perfil do usuário, tal como as tarefas envolvidas, pois Marache-Francisco e Brandier (2013) afirmam que a gamificação não pode ser efetiva a menos que ela seja baseada no entendimento de quem são os usuários e como eles utilizam o ambiente. A etapa de 
design interativo envolve a escolha das experiências que se deseja despertar no usuário. Para isso, devem-se escolher os elementos a serem utilizados, como tarefas de suporte (adaptar a interação do usuário através do uso de elementos que aumentem seus conhecimentos e habilidades, como níveis), motivação (motivar o usuário através do uso de elementos emocionais e persuasivos como pontos, medalhas e rankings) e atratividade (elementos que geram emoções positivas através da imersão, interação e uso de elementos surpresa, como as narrativas) (MARACHE-FRANCISCO; BRANDIER, 2013). Depois que esses elementos são definidos, os conceitos podem ser validados através do uso de protótipos e de testes com os usuários (MARACHEFRANCISCO; BRANDIER, 2013). Este modelo conceitual também não é específico para a área educacional.

O modelo de Simões, Redondo e Vilas (2013) propõe um framework focado nos aspectos sociais de ambientes virtuais de aprendizagem para crianças entre seis e doze anos. O principal objetivo é oferecer ferramentas dentro do ambiente que permitam o próprio professor gamificar sua disciplina. Através do modelo conceitual proposto, o professor pode escolher os elementos sociais de jogos para incentivar os comportamentos desejados, como melhorar o aprendizado no sistema (SIMÕES; REDONDO; VILAS, 2013). Esse modelo ainda está em desenvolvimento pelos autores e o próximo passo é a validação do framework através de uma pesquisa com os alunos, pais e professores (SIMÕES; REDONDO; VILAS, 2013).

Já o trabalho de Nicholson (2012) explica um modelo conceitual para criar uma gamificação significativa centrada no usuário e focada em introduzir os elementos de jogos ao invés dos elementos de pontuação. Esse modelo dá mais importância as necessidades e objetivos do usuário ao invés das necessidades da organização e, de acordo com Nicholson (2012), quando uma experiência de jogo for positiva e significativa para o usuário e essa experiência estiver bem relacionada com o objetivo original do contexto, a organização será beneficiada à longo prazo. Este modelo não é específico para a área educacional.

O estudo destes modelos tem o propósito de identificar as características importantes para a criação de um modelo conceitual para o gamificação de um ambiente virtual de aprendizagem. O Octalysis mostra duas características importantes para a gamificação: gerar uma sensação de realização e desenvolvimento para os usuários e incentivar os elementos sociais existentes em um ambiente. Marache-Francisco e Brangier (2013) afirmam que é fundamental entender quem são seus usuários e como o ambiente é usado. De acordo com Simões, Redondo e Vilas (2013), os elementos do jogo podem promover comportamentos desejados no sistema e estes comportamentos podem influenciar a aprendizagem dos alunos. E, conforme Nicholson (2012), o ambiente precisa ser centrado no usuário, pois isso envolve mais os usuários e também traz benefícios para a organização.

Diferentemente da maioria dos trabalhos relacionados, este trabalho analisou as principais características de cada modelo, e analisou-as no contexto educacional. Apesar do modelo de Simões, Redondo e Vilas (2013) estar relacionado à área educacional, duas restrições a sua utilização são expostas. Primeiro o framework é específico para crianças e em segundo lugar, o modelo ainda está em fase de desenvolvimento. Desta forma, este trabalho apresenta a proposta de um modelo conceitual para a gamificação de ambientes virtuais de aprendizagem, e sua aplicação em um ambiente real. 


\section{Modelo conceitual para gamificação de AVAs}

O modelo conceitual proposto visa auxiliar na implementação de gamificação em ambientes virtuais de aprendizagem (AVAs) existentes ou em desenvolvimento. Para isso, o modelo conceitual se divide em quatro dimensões, sendo cada uma responsável por responder uma questão sobre a gamificação criada no ambiente: por quê?, quem?, o que? e como?. Essas dimensões são mais detalhadas a seguir.

A primeira dimensão deve definir o por quê de gamificar o ambiente e quais os benefícios que a gamificação traz para o aprendizado dos alunos. O uso da gamificação, conforme explicam Simões, Redondo e Vilas (2013), permite o estímulo de alguns comportamentos no ambiente. Com base nisso, esse modelo mapeou sete comportamentos geralmente disponíveis em ambientes virtuais de aprendizagem nos quais se espera estimular os alunos, sendo: 1) Acessar as aulas, materiais, conceitos e exemplos disponibilizados pelo professor; 2) Resolver exercícios e entregar tarefas; 3) Aumentar o entendimento e desempenho no exercício; 4) Participar mais ativamente do fórum, criando e respondendo tópicos; 5) Utilizar o bate-papo; 6) Utilizar o mural de recados e; 7) Acessar o ambiente com mais frequência.

Estes comportamentos são classificados em quatro categorias diferentes: teórica (a parte teórica do sistema, como o acesso as aulas), prática (a parte prática do sistema, como a resolução e a melhora do desempenho nos exercícios), social (envolve os aspectos sociais do sistema, como uso de fórum, bate-papo e mural) e sistemática (relacionada ao sistema em si, como a quantidade de visitas e o tempo de uso do ambiente).

A segunda dimensão deve definir para quem gamificar, ou seja, quem são os atores do sistema (e.g., alunos, professores, monitores, pais). Esse modelo pretende atender os dois atores principais: os alunos (dado que o ambiente é projetado para facilitar sua aprendizagem) e os professores (pois são eles que irão incluir o conteúdo no ambiente, tal como entender as necessidades específicas e avaliar o progresso dos alunos). Além disso, o professor participa ativamente em alguns dos ambientes existentes, intervindo no conteúdo ou avaliando o desempenho dos alunos.

A terceira dimensão deve definir o que gamificar no ambiente: quais dados estão envolvidos no processo e o que precisa ser modificado para gamificar o ambiente. A quarta dimensão deve avaliar como gamificar o ambiente, definindo quais são os elementos de jogos que são utilizados para incentivar determinados comportamentos, levando em consideração o que gamificar, quem são os atores e suas características.

Para implementar pontos no ambiente, o modelo de estudante deve ser atualizado para permitir o armazenamento e o controle dos pontos obtidos pelo aluno. Essa atualização deve ser feita no banco de dados, através da criação de tabelas para armazenar as informações e também no código, que irá acionar funções de callback para serem executadas após uma determinada ação (como a conclusão de um exercício). Para implementar níveis, também é necessário modificar a base de dados para definir a forma como os níveis serão estruturados. Para implementar desafios, um modelo deve ser criado para definir as características dos desafios e também será necessário implementar uma maneira para descobrir se os desafios foram cumpridos ou não. Para isso, ferramentas como web analytics e logs podem ser utilizadas. Já rankings, medalhas e conquistas são apenas elementos visuais diretamente relacionados com os elementos como ponto e desafios, que por sua vez, devem estar sempre atualizados. A narrativa 
pode ser incluída para criar um enredo, adaptando o conteúdo educacional do ambiente. As regras são uma parte de todos os ambientes gamificados e, para utilizá-las, professores e designers devem defini-las em conjunto durante o processo de planejamento da gamificação.

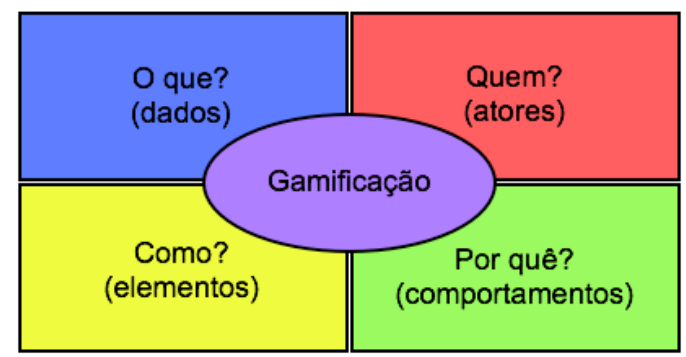

Figura 1. Quatro dimensões do modelo conceitual para gamificar ambientes virtuais de aprendizagem

Cada dimensão proposta por esse modelo se relaciona com as demais através dos sete comportamentos mapeados (i.e., acessar as aulas, resolver exercícios, melhorar o desempenho no exercício, participar do fórum, utilizar o bate-papo e o mural, além de acessar o ambiente mais frequentemente). Os comportamentos que se espera estimular no ambiente (por quê?) influenciam nos elementos de jogos que devem ser utilizados (como?) e quais perfis de alunos se espera estimular com essa mudança (quem?). Através dessa relação, é possível definir quais dados precisam ser modificados para que o ambiente seja gamificado (o que?).

Para estimular o acesso as aulas, materiais, conceitos e exemplos disponibilizados pelo professor no ambiente virtual de aprendizagem, Barata et al. (2013) explicam que elementos como pontos, níveis e rankings auxiliam no acesso as aulas e medalhas e desafios podem encorajar os alunos a ler os materiais disponibilizados e informar sobre possíveis erros encontrados nesses materiais. O’Donavan, Gain e Marais (2013) também recomendam a utilização de narrativas que se relevam conforme o acesso dos materiais. Esse comportamento envolve apenas o aluno, que opta por acessar ou não as aulas disponibilizadas.

A resolução de exercícios e entrega de tarefas pelo sistema também é comum em ambientes virtuais de aprendizagem e podem ser estimulados através de pontos, níveis, rankings, medalhas e desafios (IOSUP; EPEMA, 2014; LI et al., 2013; DENNY, 2013). Seguindo a lógica da narrativa proposta por O'Donavan, Gain e Marais (2013), a resolução de cada exercício poderia resolver um mistério exposto durante a narrativa. Esse comportamento não envolve apenas o aluno (responsável por resolver os exercícios e entregar as tarefas), mas também o professor (responsável por corrigir e avaliar as respostas dos alunos).

Além de estimular a resolução dos exercícios e entrega das tarefas, os alunos devem ser incentivados a terem um aumento do entendimento e do desempenho nesses exercícios, cometendo menos erros e melhorando seu desempenho. Para tanto, elementos como desafios e medalhas podem ser utilizados, como exemplifica o trabalho de Denny (2013), que propõe que os alunos resolvam uma lista de exercícios sem cometer erros. Esse comportamento também depende do aluno, pois cabe a ele tentar melhorar seu entendimento e desempenho nos exercícios.

A participação mais ativa no fórum pode ser incentivada por elementos como pontos, níveis, rankings, medalhas e desafios. Uma ferramenta muito bem sucedida nesse contexto é o StackOverflow, que utiliza tais elementos e recompensa as pessoas 
que criam e respondem perguntas. Esse comportamento envolve os alunos (responsáveis por participar do fórum) e o professor (que geralmente é responsável por moderar a utilização da ferramenta).

A utilização do bate-papo e do mural de recados podem ser estimulados através de medalhas e desafios, como a primeira utilização do bate-papo para conversar com um colega, ou a publicação de uma informação importante no mural são boas formas de incentivar seu uso. Nesses comportamentos, alunos e professores estão envolvidos, postando e moderando respectivamente.

Por último, um dos comportamentos mais difíceis de serem incentivados em ambientes virtuais de aprendizagem, o aumento da frequência de uso do ambiente pelos alunos. Nisso, a gamificação aplicada em todo o ambiente pode ajudar indiretamente, mas elementos como desafios e medalhas podem auxiliar, por exemplo, através da premiação de aluno que acessam o sistema diariamente em um determinado período.

\section{Aplicação do modelo no AdaptWeb}

O modelo proposto na seção 5 foi utilizado para implementar a gamificação no AdaptWeb (Ambiente de Ensino-Aprendizagem Adaptativo na $\mathrm{Web}$ ), um ambiente virtual de aprendizagem web, adaptativo e de código aberto. Esse ambiente tem uma arquitetura baseada em módulos, sendo eles: módulo de autoria (onde os materiais são organizados dentro do sistema), módulo de armazenamento (onde os dados do módulo de autoria são criados e organizados em arquivos XML), módulo de adaptação do conteúdo (adapta o material para os alunos, apresentando de forma diferente o mesmo conteúdo conforme os arquivos XML) e o módulo de interface adaptável (adapta a interface e a navegação do ambiente, de acordo com as características dos alunos) (OLIVEIRA et al., 2003).

Entre os comportamentos existentes atualmente no AdaptWeb, é possível encontrar: 1) a visualização do conteúdo inserido pelo professor, onde os alunos podem acessar o conteúdo livremente ou seguindo os pré-requisitos estabelecidos pelo professor; 2) a verificação do mural de recados, que funciona como uma forma rápida de comunicação entre os alunos e o professor e 3) o acesso ao fórum, onde os alunos podem compartilhar ideias, tirar dúvidas, entre outros. Por isso, iniciando a aplicação do modelo conceitual, o AdaptWeb foi gamificado porque deseja-se incentivar quatro comportamentos: 1) o acesso aos materiais, 2) a participação nos fóruns, 3) a utilização do mural e 4) a maior frequência de acesso ao ambiente.

Como o AdaptWeb é utilizado por turmas do curso de graduação em bacharelado em Ciência da Computação, um questionário foi feito com os alunos para identificar quem utiliza o ambiente. O questionário, Bartle Test of Gamer Psychology (1996), traça o perfil e tipo de jogador, de acordo com a definição de Bartle (1996) O questionário foi respondido por trinta e oito alunos da Universidade do Estado de Santa Catarina para identificar o tipo de jogador mais comum entre os alunos. Através deste questionário, verificou-se que a maioria dos estudantes (54\%) têm um perfil voltado para resolver quebra-cabeças e de desfrutar a vitória conseguida com muito esforço, o que se encaixa como o perfil "conquistador" definido por Bartle (1996). Com base nisso, os elementos mais utilizados para atrair esses alunos são: pontos, níveis, rankings e medalhas.

O que e como essa gamificação foi implementada no ambiente são respondidos através de elementos que geram uma sensação de progresso, de competição e de 
realização, a fim de aumentar o envolvimento e motivação. Os pontos, que são exibidos por uma barra de progresso, podem ser obtidos quando os alunos acessam o ambiente (o primeiro acesso do dia) e os materiais disponibilizados pelo professor (apenas no primeiro acesso), ao utilizar o mural de recados e o fórum, ao receber uma boa classificação do professor em uma postagem no fórum e ao completar desafios. Os níveis implementados no ambiente são incrementados conforme a quantidade de pontos que o aluno obtém (e.g., para chegar ao Nível 1 são necessários 50 pontos, para o Nível 2 são necessários 150 pontos e para o Nível 3, precisa-se obter 300 pontos). O ranking também é determinado conforme os pontos obtidos pelos alunos, apresentando os cinco alunos com a maior pontuação na página principal do ambiente. Também foram implementados alguns desafios no ambiente, como acessar diariamente o ambiente durante um determinado período, visualizar todo o conteúdo disponibilizado pelo professor, subir no ranking, utilizar o mural de recados e realizar postagens no fórum. Para cada desafio, diferentes medalhas foram criadas. Foram implementadas regras, que conduzem toda a interação dentro do ambiente. Além disso, algumas métricas (o número de acessos por aluno, a frequência de acesso, a quantidade de acessos ao material disponibilizado e a participação no fórum) foram implementadas para visualizar o que os alunos realizam no sistema e mensurar seu engajamento.

A Figura 2 demonstra a página do Perfil do Aluno, onde diferentes elementos de gamificação foram implementados. É possível visualizar os pontos obtidos, nível atual, medalhas conquistadas e o ranking.

AdaptWeb
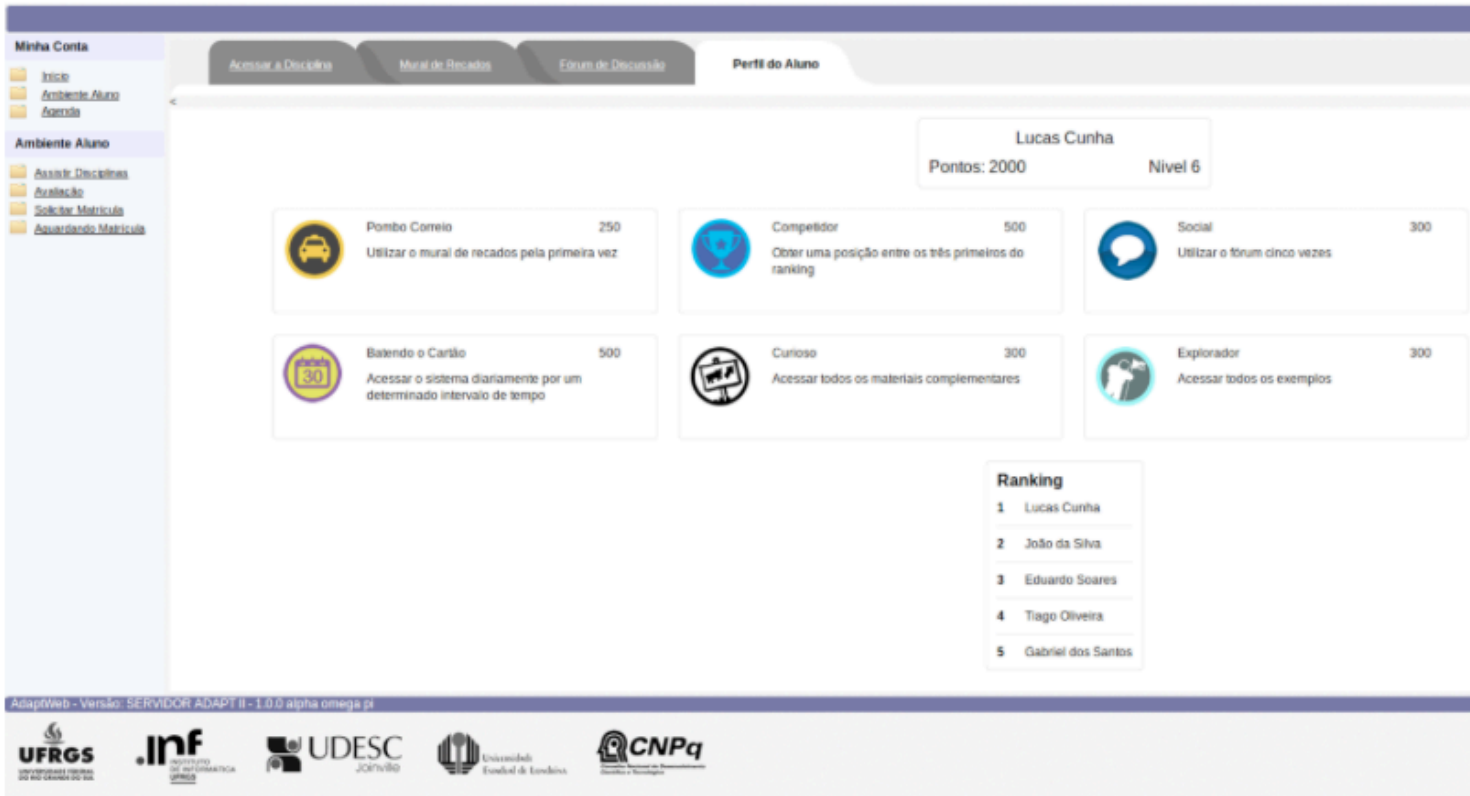

Figura 2. Processo de gamificação no AdaptWeb através do modelo conceitual

Além disso, foram implementados elementos para engajar diferentes tipos de jogadores: os 'achievers', através de pontos e níveis, os 'socializers' através das ferramentas de comunicação como fórum e mural, 'explorers', com desafios ocultos, onde são conquistadas as medalhas, e os 'killers', através do ranking. Atualmente, o modelo conceitual utilizou os tipos dos jogadores segundo a definição de Bartle (1996), mas pretende-se futuramente adaptar a gamificação para cada aluno através de suas 
características pessoais, cognitivas e culturais, dando enfoque aos elementos que tendem a melhor motivar cada aluno.

\section{Conclusão}

A gamificação é uma ferramenta que tem sido utilizada para auxiliar a motivar e engajar as pessoas no alcance de um objetivo específico. No ambiente educacional, a gamificação permite que o aluno se divirta enquanto aprende, tornando o processo de ensino-aprendizagem mais engajador e motivador para alunos e professores. Para implementar o processo de gamificação, existem diversos frameworks que explicam quais técnicas devem ser utilizadas, considerando diferentes tipos de usuários. $\mathrm{O}$ modelo conceitual proposto nesse artigo explica o que, como, o por quê e para quem realizar a gamificação de um ambiente virtual de aprendizagem. Ele auxilia na aplicação da gamificação em ambientes virtuais de aprendizagem, considerando o conteúdo do ambiente, as tarefas nele disponíveis e as características dos alunos que o utilizam. Esse modelo foi demonstrado através de sua aplicação no AdaptWeb, um ambiente virtual de aprendizagem adaptativo. Ainda não foi realizado um experimento para validar a implementação.

Entre os trabalhos futuros, pretende-se avaliar outras características dos alunos, tal como características pessoais, preferências, estilos cognitivos de aprendizagem e questões culturais, mapeando tais características e adaptando a interface para dar enfoque diferenciado a cada elemento de acordo com o aluno que está utilizando o ambiente.

\section{Referências}

ALMALIKI, M.; JIANG, N.; ALI, R.; DALPIAZ, F. "Gamified culture-aware feedback acquisition". In: 2014 IEEE/ACM 7th International Conference on Utility and Cloud Computing (UCC), p. 624-625, London, IEEE, 2014.

ALMALIKI, M.; NCUBE, C.; ALI, R. "The design of adaptive acquisition of users feedback: An empirical study". In: IEEE Eighth International Conference on Research Challenges in Information Science, p. 1-8, Marrakesh, IEEE, 2014.

BARATA, G.; GAMA, S.; JORGE, J.; GONÇALVES, D. "Engaging engineering students with gamification". In: 5th International Conference on Games and Virtual Worlds for Serious Applications, p. 1-8, Bournemounth, IEEE, 2013.

BARTLE, R. "Hearts, Clubs, Diamonds, Spades: Players who suit MUDs". In: Journal of MUD Research, 1996.

BARTLE TEST OF GAMER PSYCHOLOGY. Disponível em: http://www.gamerdna.com/quizzes/bartle-test-of-gamer-psychology. Acesso: 28 abr. 2015.

BBVA INNOVATION EDGE. "The fun way to engage". In: Gamification: The business of fun, p. 12-22, Madrid, BBVA Innovation Center, 2012.

BISSOLOTTI, K.; NOGUEIRA, H. G.; PEREIRA, A. T. C. "Potencialidades das mídias sociais e da gamificação na educação a distância". In: RENOTE - Revista de Novas Tecnologias na Educação, v. 12, no. 2, 2014.

BURKE, B. Gamification Primer: Life becomes a game. Gartner, 2011.

BURKE, B. Gamify: How gamification motivates people to do extraordinary things. Bibliomotion, 2014.

CHOU, Y. K. Octalysis: Complete Gamification Framework. Yu-Kai Chou \& Gamification, 2013.

CODISH, D.; RAVID, G. "Personality based gamification-Educational gamification for extroverts and introverts". In: Proceedings of 9th Chais Conference for the Study of Innovation and Learning Technologies, p. 36-44, Raanana, The Open University of Israel, 2014. 
DENNY, P. "The effect of virtual achievements on student engagement". In: Proceedings of the SIGCHI Conference on Human Factors in Computing Systems, p. 763-772, New York, Association for Computing Machinery, 2013.

DETERDING, S.; DIXON, D.; KHALED, R.; NACKE, L. "Gamification: Toward a definition". In: Proceedings of the 2011 Conference on Human Factors in Computing Systems, Association for Computing Machinery, 2011a.

DETERDING, S.; DIXON, D.; KHALED, R.; NACKE, L. "From game design elements to gamefulness: defining 'gamification"”. In: Proceedings of the 15th International Academic MindTrek Conference: Envisioning Future Media Environments, p. 9-15, Tampere, Association for Computing Machinery, 2011b.

DRAPER, S. W. "Analysing fun as a candidate software requirement". In: Journal of Personal Technologies, volume 3, issue 3, p. 117-122, Springer-Verlag, 1999.

FARDO, M. F. "A gamificação aplicada em ambientes de aprendizagem”. In: RENOTE Revista de Novas Tecnologias na Educação, v. 11, no. 1, 2013.

HÄGGLUND, P. Taking gamification to the next level. Umea University, 2012.

IOSUP, A.; EPEMA, D. "An experience report on using gamification in technical higher education". In: Proceedings of the 45th ACM technical symposium on Computer science education, p. 27-32, Association for Computing Machinery, 2014.

JOHNSON, L.; ADAMS, S.; CUMMINS, M.; ESTRADA, V.; FREEMAN, A.; LUDGATE, H. The NMC Horizon Report: 2013 Higher Education Edition. New Media Consortium, 2013.

KLOCK, A. C. T.; CARVALHO, M. F.; ROSA, B. E.; GASPARINI, I. "Análise das técnicas de Gamificação em Ambientes Virtuais de Aprendizagem". In: RENOTE - Revista de Novas Tecnologias na Educação, v. 12, no. 2, 2014.

LI, C.; DONG, Z.; UNTCH, R. H.; CHASTEEN, M. "Engaging computer science students through gamification in an online social network based collaborative learning environment". In: International Journal of Information and Education Technology, v. 3, n. 1, p. 72-77, 2013. MALONE, T. W. "Heuristics for designing enjoyable user interfaces: Lessons from computer games". In: Proceedings of the 1982 Conference on Human Factors in Computing Systems, Association for Computing Machinery, 1982.

MARACHE-FRANCISCO, C.; BRANGIER, E. "Process of gamification. From the consideration of gamification to its practical implementation". In: The Sixth International Conference on Advances in Human oriented and Personalized Mechanisms, Technologies, and Services, p. 126-131, Venice, IARIA, 2013.

NICHOLSON, S. "A User-Centered Theoretical Framework for Meaningful Gamification”. In: Games, Learning and Society Conference, Madison, University of Wisconsin, 2012.

O'DONAVAN, S.; GAIN, J.; MARAIS, P. "A case study in the gamification of a universitylevel games development course". In: South African Institute for Computer Scientists and Information Technologists Conference, Association for Computing Machinery, 2013.

OLIVEIRA, J. P. M. D.; BRUNETTO, M. A. O. C.; PROENÇA JUNIOR, M. L.; PIMENTA, M. S.; RIBEIRO, C. H. F. P.; LIMA, J. V. D.; FREITAS, V. de; Marçal, V. S. P.; Gasparini, I.; AMARAL, M. A. "AdaptWeb: um ambiente para ensino-aprendizagem adaptativo na Web". In: Educar em revista, p. 175-197, Curitiba, NESP, 2003.

PEDRO, L. Z.; LOPES, A. M.; PRATES, B. G.; VASSILEVA, J.; ISOTANI, S. "Does Gamification Work for Boys and Girls? An Exploratory Study with a Virtual Learning Environment". In: 30th ACM/SIGAPP Symposium On Applied Computing, Salamanca, Association for Computing Machinery, 2015.

SIMÕES, J.; REDONDO, R. D.; VILAS, A. F. "A social gamification framework for a k-6 learning platform". In: Journal of Computers in Human Behavior, volume 29, issue 2, p. 345-353, Elsevier Science Publishers, 2013.

WERBACH, K.; HUNTER, D. For the win: How game thinking can revolutionize your business. Wharton Digital Press, 2012.

ZICHERMANN, G.; LINDER, J. Game-based marketing: inspire customer loyalty through rewards, challenges, and contests. Hoboken, John Willey \& Sons, 2010. 